\title{
A Path for Tracking Funded Research and Compliance
}

\section{by Howard Ratner and Susan Spilka}

\section{$\mathrm{T}$} o better understand how CHORUS [1] is creating a future where the output flowing from funded research is easily and permanently discoverable, accessible, and verifiable, this article will share the story of its origins, present an overview of its services, and look at what's in the pipeline. Working closely with organizations from all corners of the scholarly community, CHORUS is helping to establish new foundational standards, metrics, and metadata. This work is enabling CHORUS to introduce new and expanded capabilities-notably, tracking research datasets and integrating institutional identifiers-that support the continuing evolution of a sustainable and connected open-science infrastructure.

\section{Taking Shape}

As founding CHORUS member Fred Dylla (American Institute of Physics, 2007-2015) and Jeffrey Salmon (U.S. Department of Energy Office of Science, 2002-2017) tell the story [2], CHORUS was conceived on the February 2013 day that the U.S. White House Office of Science and Technology Policy (OSTP) issued a public access directive. That memorandum mandated new requirements for major U.S. funding agencies to set goals and timelines to make the published output of the research they funded freely accessible to the public [3]. The same day, a group of industry leaders met under the aegis of the Association of American Publishers (AAP) to discuss the formation of an organization that could assist U.S. federal agencies in their implementation of the OSTP directive. They wanted to provide a flexible and efficient route to compliance. Central to their objectives was avoiding additional researcher burdens and incorporating the process within existing publishing workflows.

In short order, the publishers mapped out the strategy for the sustainable path to public access that became CHORUS, continuing their earlier collaboration with the Department of Energy (DOE), and the National Science Foundation (NSF) that began after the America Competes Act became law in the U.S. in 2010. This earlier group wanted to utilize the relatively new Crossref funding-agency identifier called FundRef, based on the Digital Object Identifier (DOI), the underlying data in scholarly literature that enables online reference linking. Now known as the Crossref Funder Registry [4], the tagging system they developed efficiently and economically identifies the funders who support the research reported in all articles receiving DOIs. NASA and Wellcome Trust joined the effort to help design the new persistent identifier (PID). For the solution to work, the founding CHORUS group determined that it had to also provide access to the open content and information about license terms, and ensure archival longevity to the research output. That is why CHORUS came to exist.

The initiative was incorporated as CHOR, Inc., a non-profit membership organization. Its founding mission, which remains largely unchanged, is "to support and promote open access to and continued availability of publications reporting on funded research by leveraging new and existing technologies used throughout the publishing and scholarly communications community." What has changed, in response to the needs of the scholarly community, is that the CHORUS mission now supports and promotes open access and continued availability of the underlying datasets, in addition to the articles. Based on the public benefit of that work and the rigor of its governance practices, the organization was designated as a 501(c)(3)-a tax-free category for charitable non-profit organizations-by the U.S Internal Revenue Service.

Just as it efficiently leverages the technical infrastructure, CHORUS relies on the active engagement of a members-elected Board of Directors on its committees and task forces to advance its mission and objectives. A small team, led by Executive Director Howard Ratner, manages its operations. The Board membership represents key constituencies, including society and commercial publishers, publishing service companies, industry organizations, and academic libraries from major research institutions. Other stakeholders in the scholarly community also influence the operations of CHORUS through participation in working groups and advisory committees. Crossref remains an important CHORUS service provider. CHORUS also partners with CLOCKSS and Portico to ensure the archiving and preservation of research papers, and with other industry initiatives such as ORCiD, DataCite, SCHOLIX, Metadata 2020, and the Research Organization Registry.

\section{Adding Value}

CHORUS hit the ground running because of the substantial investments made over the years by the scholarly publishing community in an open, interoperable, and scalable research communications infrastructure. This framework set CHORUS apart from the start. As American Physical Society Publisher Matthew Salter explained, "There's an important process that goes on between discovery in a laboratory or in an academic setting, and communicating that research to other researchers and the public. CHORUS coalesces best 


\section{A Path for Tracking Funded Research and Compliance}

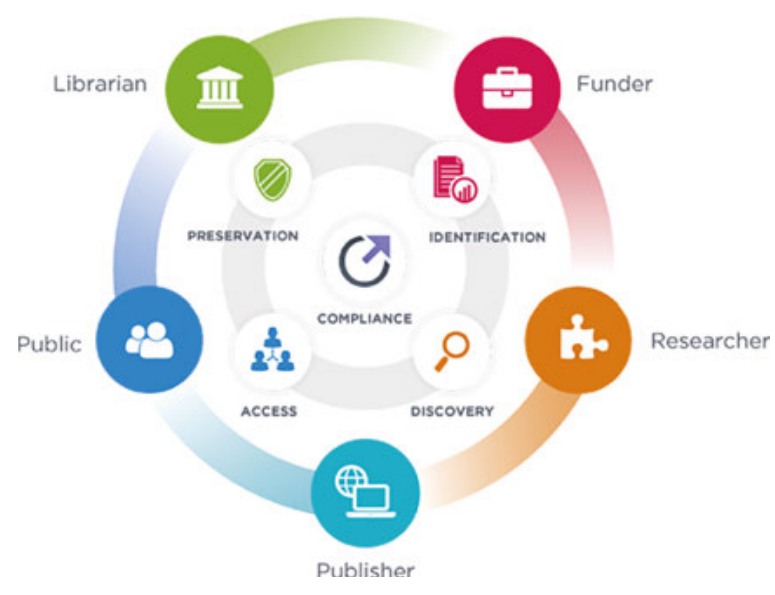

practices, streamlines workflows, and aligns the interests of researchers, publishers, government agencies, research officers, and librarians."

CHORUS currently counts as members 42 society and commercial publishers (Publisher Members) who collectively publish over 11,400 journals-the vast majority of articles reporting on publicly funded research. Six related businesses and organizations that provide essential technology and services participate as Affiliate Members. To participate, members follow the Publisher Implementation Guide outlined and updated by the Technical Working Group. Members have commented that onboarding with CHORUS propelled them to implement metadata best practices that they hadn't done previously, and that collaboration with other working group members was helpful to their internal workflows.

Self-sustaining through memberships and fees, CHORUS offers a growing portfolio of services for publishers, funders, and institutions. Ten U.S. government funding agencies, and one in Japan, are currently working with CHORUS as part of their public-access plans. Funders and institutions both play a key advisory role in CHORUS development and are beneficiaries of the services it provides. Their engagement has been a factor in CHORUS' success in broadening open access, clearing a collective path to compliance, and providing a tool to assess the return on research investments. Facilitating coordination among publishers, institutions, and funders is an important CHORUS value-add.

\section{How CHORUS Works}

By weaving existing systems and services together with new ones, CHORUS has been able to quickly facilitate an efficient, data-driven solution that is interoperable across the spectrum of publishing models, platforms, and portals. As a result of its distributed access approach, there are now paths to compliance covering research on publication sites as well as on agency portals and repositories. By directing users to the publication sites where article-level metrics accrue, CHORUS helps to enable more accurate assessments of research impact and return on investment. The underlying metadata make research outputs more discoverable.

To initiate CHORUS' services, authors simply have to identify their funding sources when submitting a paper for publication with a participating publisher. That action associates the article with Crossref Funder Registry identifiers. Publisher submission workflows also gather other identifying information and metadata that become part of the record and help to determine when and which version of an article will be made openly available and other functions, such as researcher identity disambiguation with their ORCID identifier.

Publishers deposit article metadata records to the Crossref database, where they are assigned DOIs. CHORUS continuously ingests and indexes the metadata associated with those records, audits for open accessibility (tracking whether the content will be open immediately on publication or after a designated embargo period), availability of reuse licenses, archival and preservation arrangements, public grant information, author ORCID ID records, datasets associated with the published article, and links from funder open or public access repositories. CHORUS then analyzes the audited data and creates dynamic information dashboards, reports, and APIs of key performance indicators that enable funders, publishers, and institutions to monitor and report on compliance with open-access requirements.

The initial CHORUS offerings were its dashboards and associated reports for monitoring and tracking publisher contributions. The first dashboards released were for funder partners, which continue to be publicly accessible on the CHORUS website. The records contain links to the content on publisher site(s), but metadata can be exported to agency portals (e.g., National Science Foundation) and publisher platforms through the CHORUS open API. Other valuable resources that CHORUS provides include a tool to find agency funder IDs and a detailed compendium of funding agency public access plans.

Early on CHORUS also introduced dashboards and reports for Publisher Members, providing a onestop resource for tracking access status and funder compliance requirements for the journal articles they publish. While research grant holders are ultimately 


\section{A Path for Tracking Funded Research and Compliance}

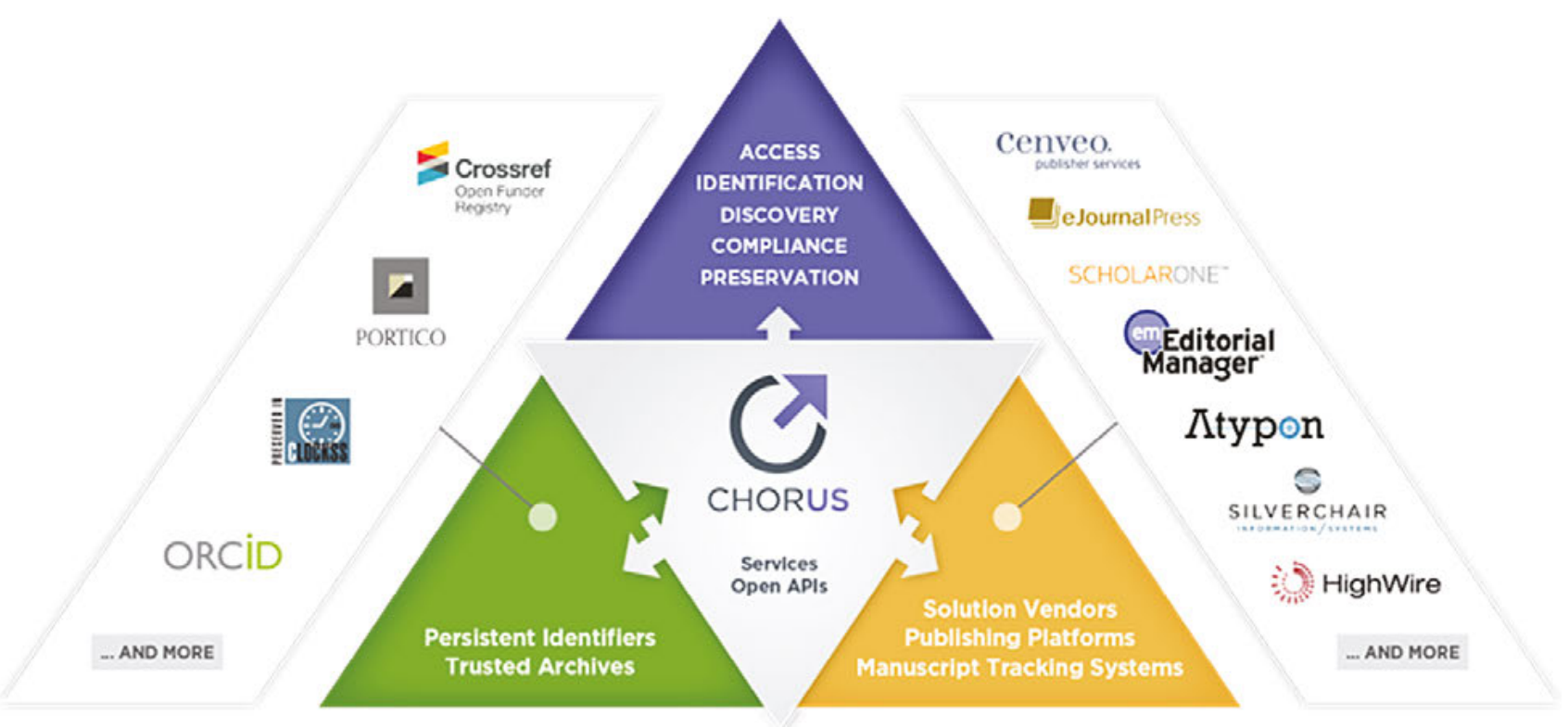

accountable for compliance, embedding the verification process in the manuscript submission workflowwithout adding tasks for author(s)-serves the greater good. As Rockefeller University Press Executive Director Dr. Susan King articulated, "Given the importance of government-funded researchers to RUP's success, we believe we have an obligation to give something back to them, as well as the agencies and the public. As an added bonus, implementation of the CHORUS workflow has revealed that the breadth of funding for RUP published research is much, much wider than we originally thought." [5]

\section{Piloting New Territories}

U.S. Academic Institutions-Research institutions have a fiduciary interest in making sure that their faculty researchers comply with funder requirements. Yet there is considerable inconsistency in how they meet the challenge. To collaborate on the best approach to monitoring faculty research output and increasing compliance rates, the CHORUS Institution Dashboard pilot program was launched in 2017, with seven Publisher Members taking part. The initial pilots allowed CHORUS to better understand and respond to the challenges institutions face, as their experience with the first participants illustrates:

University of Florida conducted a survey that found faculty put a high priority on the widest dissemination of their research publications, but could not devote the necessary time and resources. The UF Libraries saw CHORUS as a way to ease mandate compliance without further burdening researchers. The CHORUS Institution Dashboard was employed as a central portal for identification of faculty articles from a variety of publishers, forming the corpus of UF scholarship metadata to which its institutional repository (IR@UF) linked. UF developed its own email notification process tied to the CHORUS Dashboard deposit data, to keep faculty and administrators informed about compliance milestones. According to Dr. Sherry Larkin, UF Associate Dean for Research, "The CHORUS dashboard and the availability of downloadable .csv files have the potential to ease the time and financial burden of this annual process on our organization."

University of Denver faculty researchers reported they were unaware of or confused by funder mandates, what version (author accepted manuscript, publisher version of record, etc.) should be deposited, and which archives would satisfy compliance. Compliance wasn't "high on the priority list of many researchers at the University," noted UD Libraries Associate Dean Jack Maness. He explained that the administration "don't want it to be. Their [researchers] role is to do research. Ours is to ensure that the results of that research are as widely available as possible, now and for future generations. As librarians, we want to do what we can to help shoulder what could be considered an administrative burden, because it aligns with our values as a profession: providing access to knowledge that benefits humanity." By using dashboard data and services in conjunction with their own internal data, UD librarians were able to develop an automated solution to monitor public accessibility to the University's publicly funded research.

Both pilot participants found the CHORUS Institution Dashboard so effective at helping monitor 


\section{A Path for Tracking Funded Research and Compliance}

compliance of their federally funded research that they signed an agreement for services in 2018. CHORUS recently launched the new service and begun to sign on academic libraries as CHORUS Institution Dashboard subscribers.

\section{CHORUS in Japan}

With the significant growth in research output in the Asia-Pacific region, it was logical for CHORUS to seek international expansion in this region as a next step. Australia and Japan over many years have sustained their prolific presence in the global top-ten economies by research output as measured by the annual output of published articles; in 2016 CHORUS started pilot projects in both countries. The Australian Research Council (ARC) partnered with La Trobe University; and Japan Science and Technology Agency (JST) with Chiba University. Both agencies have clear OA compliance needs with the two universities having significant research profiles.

Encouragingly the pilot in Japan led to JST partnering and Chiba subscribing to the CHORUS services - the first relationships outside the U.S., and they are both doing well four years later. CHORUS collaborated with JST to improve Japanese funder information in the Crossref Funder Registry and to educate grantees on how to reference JST grants, and worked with some member publishers to improve metadata. As a result, over time they have seen a much higher capture of journal articles associated with JST funding. Dr. Yasushi Ogasaka of JST, who wrote Landscape of Open Science in Japan [6] was a speaker at the $\mathrm{CHO}$ RUS Forum on Open Access Policies and Global Compliance in a Global Context on 30 July 2020.

Working through some technical challenges with Chiba, CHORUS established the author affiliation reports that are now a standard offering for all institutions. This enabled Chiba to develop a workflow that uploads links to OA published work from their research programs for access by the wider Chiba community from their institutional repository as explained in the Japan National Library publication Current Awareness (translation) [7].

Not only does Chiba have a more comprehensive picture on research output funded from within Japan but "we also value the references to funders outside of Japan in the CHORUS data where Chiba University researchers have been able to access funding from overseas and where they have collaborated with researchers in other countries," as Professor Hiroya Takeuchi, Vice President and University Librarian, explained.

The relationships with JST and Chiba since 2016 have been reviewed and highlighted at a number of open science and library events from the end of 2017 through to 2020, most recently with a joint JST/CHORUS workshop in February. Keio University, National Institute for Materials Science, and RIKEN and Tohoku Gakuin University subscribed in 2019 with National Institute for Environmental Studies of Japan and Tokyo Metropolitan Institute of Medical Science joining in 2020.

\section{Taking it to the Next Level}

Professor Takeuchi touched on something important when he commented, "we expect that CHORUS could be our best route to OA, especially since they began to offer research datasets for integration. We were delighted when CHORUS began to work with SCHOLIX, allowing us to upload links to research datasets in our IR." What he was referring to was CHORUS' important new capability: tracking datasets and related code and connecting them to the related publications via the SCHOLIX framework, employing metadata from DataCite and other sources.

The work is an outgrowth of collaboration with organizations (including Enabling FAIR Data Repositories, DataCite, Research Data Alliance, Crossref Funder Registry, and ORCID) that are developing critically needed standards to support researchers with data management requirements, and funding bodies with compliance monitoring. It showcases how CHORUS has continued to find ways to develop better interoperability between existing systems, serve compliance with evolving funder mandates, and further the discovery of and access to funded research outputs.

CHORUS has also created a centralized index of its member publishers' data availability policies with links to the policies on the publisher's site. (https://www. chorusaccess.org/resources/chorus-for-publishers/ publisher-data-availability-policies-index/). CHORUS plans to integrate the open identifier for institutions now being developed by the Research Organization Registry. While the main focus for now is identification and links to relevant datasets, future efforts could focus on more actively engaging researchers.

Another recent enhancement has been the significant expansion of the published content CHORUS covers. Until last year, CHORUS tracked published articles reporting on research funded by its funder partners. But in 2019, CHORUS began a project to identify all content assigned DOIs that reported on research funded by all agencies worldwide with a funder ID. After the release of the second phase in June 2020, CHORUS now tracks more than 2.4 million research artifacts, 


\section{A Path for Tracking Funded Research and Compliance}

and has identified more than 260,000 DOIs associated with its publisher members as openly accessible. These totals will continue to rise as newly published and previously uncovered items are identified.

\section{0 \& Beyond}

Seven years ago, CHORUS set out to advance sustainable public access via an approach of leveraging existing open infrastructure and working closely with stakeholders. The strategy worked, delivering flexible, neutral, and interoperable solutions as policy implementation progressed and evolved around the world. Measurable progress has been made:

In 2013, funders were rarely identified in articles. Finding relevant datasets was a wish-list item. Reuse licenses were impossible to find. Funders rarely intersected with publishers and articles reporting on funder research were rarely publicly accessible.

Now in 2020, funder IDs are part of publication workflows. Identifying relevant datasets has begun in earnest. Reuse licenses are easier to find. Articles reporting on funder research are much more publicly accessible. Public access and license workflow issues are being enhanced. It is significant that 13 publishers, academic institutions and organizations named CHORUS as a solution for linking data and grant information to publications and facilitating public access to content in their responses to the OSTP RFI on Public Access to Peer Reviewed Scholarly Publications, Data, and Code Resulting from Federally-Funded Research.

One of the most important CHORUS achievements is the collective and transparent way for funders, institutions, publishers, and researchers to work together. To that end, the CHORUS Forum on Open Access Policies and Compliance in a Global Context, https://www. chorusaccess.org/events/forum-on-open-accesspolicies-and-compliance/, postponed from March due to the pandemic, was rescheduled to 30 July 2020 as an open virtual event. Funders, societies, publishers, and academic institutions came together to examine and discuss the current and future issues about access to publications and data reporting on funded research. Attendees learned about the state of play at government agencies globally regarding access policy, as well how the flow of funding impacts universities, and what Research Offices are doing about the challenges of compliance. In addition, CHORUS co-hosted a free webinar, with the STM and the Center for Open Science, Towards a US Research Data Framework, on 17 Sep 2020.

CHORUS will continue to look for ways to reinforce funders, publishers, and institutions around the world as their needs and capacities to drive open science continue to grow.

Howard Ratner (https://orcid.org/0000-0002-2123-6317) is CHORUS Executive Director, and Susan Spilka, Strategic Communications Director at TBI Communications (https://orcid.org/0000-0002-0196-558X)

\section{References}

1. CHORUS, https://www.chorusaccess.org

2. H. Frederick Dylla and Jeffrey Salmon, Learned Publishing, first published 27 March 2020, https://doi. org/10.1002/leap.1298.

3. John P. Holdren, Increasing Access to the Results of Federally Funded Scientific Research, OSTP, 22 February 2013; https://obamawhitehouse.archives.gov/ sites/default/files/microsites/ostp/ostp_public_access_ memo_2013.pdf

4. Crossref Funder Registry, http://crossref.org/fundref/

5. Sara Girard, The Small But Mighty Publisher, CHORUS News, 7 Dec 2018; https://www.chorusaccess.org/thesmall-but-mighty-publisher/

6. Yasushi Ogasaka, Landscape of Open Science in Japan, CHORUS paper, Dec 2019, https://www.chorusaccess. org/landscape-of-open-science-in-japan/

7. Nanako Takahashi and Akiko Chiba, Evaluation of the CHORUS Dashboard Service through Initiatives in Chiba University Library, CHORUS News, 18 Feb 2020; https://www.chorusaccess.org/chorus-dashboardservice-and-initiatives-at-chiba-university-library/ 